\title{
A Comparison of the Antioxidant Properties and Total Phenolic Content in a Diatom, Chaetoceros sp. and a Green Microalga, Nannochloropsis sp.
}

\author{
Su-Hua Goh \\ Laboratory of Molecular Biomedicine \\ Institute of Bioscience, Universiti Putra Malaysia \\ 43400 Serdang, Selangor, Malaysia \\ E-mail: gshstar@hotmail.com \\ Fatimah Md Yusoff \\ Laboratory of Marine Science and Aquaculture \\ Institute of Bioscience, Universiti Putra Malaysia \\ 43400 Serdang, Selangor, Malaysia
}

Tel: 60-3-8947-2111 E-mail: fatimah@ibs.upm.edu.my

Su-Peng Loh (Corresponding author)

Department of Nutrition and Dietetics

Faculty of Medicine and Health Sciences, Universiti Putra Malaysia

43400 Serdang, Selangor, Malaysia

Tel: 60-3-8497-2432Ｅ-mail: sploh@medic.upm.edu.my

This work was supported by Agri-Science Fund Grant, from the Ministry of Agriculture, Malaysia (Project No. 5450384).

\begin{abstract}
The aquaculture industry commonly makes use of microalgae as live feed. Chaetoceros sp., a diatom, and Nannochloropsis sp., a unicellular green microalga, have both been reported to contain a substantial amount of polyunsaturated fatty acids and carotenoids. Their potential as natural sources of antioxidants has gained recent attention. This study focuses on determining the antioxidant properties of the different solvent extracts, namely hexane, dichloromethane, chloroform and methanol, from both microalgae. The evaluation of antioxidant capacities was done by Folin-ciocalteu, 1,1-diphenyl-2-picrylhydrazyl radical-scavenging, metal chelating, nitro-blue tetrazolium reduction and ferric-reducing antioxidant power assay. Results showed that the non-polar solvent extracts from the diatom were highest in antioxidant power, whereas both polar and non-polar solvent extracts of green microalgae showed good antioxidant potential. In general, Chaetoceros sp. had higher antioxidant capacities than Nannochloropsis sp. This study suggests that different solvent extracts contain different potential antioxidant compounds able to scavenge different types of free radicals.
\end{abstract}

Keywords: Chaetoceros sp., Nannochloropsis sp., Marine microalgae, Antioxidant, Total phenolic contents

\section{Introduction}

In recent years, marine microorganisms have been the subject of much interest related to their neutraceutical properties, as well as their use as alternative food sources, as reported in Africa, India and Mexico (Aoronson \& Dubinsky, 1982). Nutrient-rich marine microalgae offer a promising field of study, to uncover new applications for these uses. Indeed, the ability of marine microalgae to synthesize secondary metabolites, a quality unlikely to be found in terrestrial organisms, increases its potential to become a new biomedical resource (Katsumi, 1997). Chaetoceros sp., a diatom in the class of Bacillariophycae, has gained attention due to its valuable polyunsaturated fatty profile. Furthermore, it is widely cultivated as live feed for bivalves and crustaceans (Tolga, 
Yaşar \& Şevket, 2003). Nannochloropsis sp., on the other hand, is a unicellular green alga, spherical in shape, with diameter of about $2-5 \mu \mathrm{m}$, belonging to the eustigmatophyceae class. It plays an important role in the food chain system, and is also commonly used as live feed; thus, it is widely cultivated in fish hatcheries and shrimp farms (Gwo, Chiu, Chou \& Cheng, 2005).

Lately, more and more marine microalgae have been suspected of having strong antioxidant properties, including Fucus vesiculosus (Antonio, Isabel, Raquel \& Fulgencio, 2001), Ecklonia cava (Yasantha, Kim \& Jeon, 2006), Petalonia binghamiae (Takashi, Tomoko \& Sayuri, 2006) and Scytosiphon lomentaria (Takashi, Makiko, Tomoko \& Yoko, 2004). An antioxidant is generally defined as any substance that effectively prevents or delays the adverse effects caused by free radicals, even when the amount of the antioxidant substance is less than the substance to be oxidized (Halliwell \& Gutteridge, 1999). Normally, aerobic metabolisms produce free radicals, a common necessity, but one which can be considered abnormal when the amounts are out of the normal range. External causes, such as radiation, cigarette smoke and pollutants, may also indirectly produce free radicals (Christophersen, Jun, Jorgensen \& Skibsted, 1991). Free radicals can be produced during every step in a chain reaction; thus, it is important for an antioxidant to prevent the chain initiation step by scavenging the initiator radical (Saha, Lajis, Israf, Hamzah, Khozirah, Khamis et al., 2004).

In this present study, both Chaetoceros sp. and Nannochloropsis sp. were evaluated for their antioxidant capacities using different solvent extracts with differing polarities. These solvents were hexane, dichloromethane, chloroform and methanol extract, tested against different types of radicals by Folin-ciocalteu, 1,1-diphenyl-2-picrylhydrazyl (DPPH) radical-scavenging, ferrous reducing, ferrous ion chelating, nitro-blue tetrazolium (NBT) reduction, ferric-reducing antioxidant power (FRAP) and ABTS radical cation decolorization assay.

\section{Materials and method}

\subsection{Chemicals}

All solvents were of analytical grade, and chemicals including hydrochloric acid, hypoxanthine, xanthine oxidase, nitroblue tetrazolium chloride and EDTA were purchased from Merck (Darmstadt, Germany). All other chemicals of analytical grade were purchased from Sigma-Aldrich (MO, USA), unless otherwise noted.

\subsection{Marine microalgae cultivation and sample preparation}

Sources of two microalgae, Chaetoceros sp. and Nannochloropsis sp., were provided by the Marine and Aquaculture Laboratory of Institute Bioscience, Universiti Putra Malaysia. Microalgae were cultivated in artificial seawater with a salinity of 30 ppt. Conway media (a main mineral solution, a trace metal solution, a silicate solution and a vitamin solution) were added as nutrient sources for growth. The entire growth medium was adjusted to $\mathrm{pH} 8$ and the microalgae were grown with aeration in illuminated conditions at room temperature. They were harvested after 5-7 days (the stationary phase) and centrifuged (using Hitachi high Speed Refrigerated Centrifuge Models CR22G II/CR $21 \mathrm{G} \mathrm{II}$ ) at $8000 \mathrm{rpm}$ and $23^{\circ} \mathrm{C}$, for 10 minutes for the green microalgae or 20 minutes for the diatom. Then, the harvested microalgae were rinsed with either distilled water for the green microalgae or ammonium sulfate for the diatom. The preparation was then centrifuged again and freeze-dried (using Labconco 77540) for long-term storage.

\subsection{Sample extract preparation}

Two grams of freeze-dried powder was soaked in $500 \mathrm{ml}$ of either hexane, dichloromethane, chloroform or methanol, and was shaken for 24 hours, then filtered through filter paper. The solvent was then evaporated in a rotary evaporator (EYELA N-1000S-WD) at $35^{\circ} \mathrm{C}$ until dried, and kept at $-20^{\circ} \mathrm{C}$ until further analysis was begun.

\subsection{Antioxidant evaluation assays}

\subsubsection{DPPH Radical Scavenging Assay}

The total antioxidant capacity was evaluated using a stable $\mathrm{DPPH}^{+}$radical following the Tarozzi, Marchesi, Cantelli-Forti \& Hrelia (2004) method, with a slight modification of the concentration and volume used. A sample solution was added into each well for a final concentration of $1 \mathrm{mg} / \mathrm{ml}$, with an additional $100 \mu \mathrm{l}$ of methanol added as a medium for the reaction. Finally, $5 \mu 1$ of DPPH solution $(2.5 \mathrm{mg} / \mathrm{ml})$ was added. All the results were expressed as $\mu \mathrm{mol}$ Trolox Equivalent (TE) per gram of dry weight of the microalgae.

\subsubsection{Superoxide Anion Scavenging Assay}

Measurement of the superoxide radical scavenging was done by nitroblue tetrazolium chloride (NBT) assay. This assay was conducted following the Lee, Yoon, Kim and Lim (2004) method, with slight modifications. 
Solution A containing $0.2 \mathrm{mM}$ Hypoxanthine (in potassium hydroxide), $0.1 \mathrm{mM}$ NBT (in $50 \mathrm{mM}$ potassium phosphate buffer with a $\mathrm{pH}$ of 7.5), $0.05 \mathrm{mM}$ EDTA solution and $50 \mathrm{mM}$ potassium phosphate buffer with a $\mathrm{pH}$ of 7.5 was prepared in a ratio of $4: 2: 1: 13$. Solution B contained $0.08 \mathrm{U} / \mathrm{ml}$ of xanthine oxidase that was diluted with $50 \mathrm{mM}$ potassium phosphate buffer. Solution $\mathrm{C}$ was prepared in $1 \mathrm{~N} \mathrm{HCl}$. The reaction started when $20 \mu \mathrm{l}$ of the sample solution was added to $100 \mu \mathrm{l}$ of solution A followed by solution B. Then, the mixture was incubated in a $37^{\circ} \mathrm{C}$ incubator for 20 minutes. Then, solution $\mathrm{C}$ was added to terminate the reaction before absorbance was measured at $540 \mathrm{~nm}$. All the results were expressed as $\mu \mathrm{mol}$ Trolox Equivalent (TE) per gram dry weight of the microalgae.

\subsubsection{Ferric-Reducing Antioxidant Power (FRAP) Assay}

The FRAP assay was carried out as suggested by Omidreza, Antonio, Rita, Giancarlo \& Luciano (2005), with a modified concentration. FRAP reagents were freshly prepared for each measurement by mixing $10 \mathrm{mM} 2,4,6-$ tripyridyl triazine (TPTZ) in $40 \mathrm{mM}$ of $\mathrm{HCl}, 0.3 \mathrm{M}$ of pH 3.6 acetate buffer and $20 \mathrm{mM}$ ferric chloride (from Acros organics, Belgium) in double-distilled water in a ratio of $1: 10: 1$. A volume of $180 \mu \mathrm{l}$ of $37^{\circ} \mathrm{C}$ pre-warmed FRAP reagent was added with $20 \mu \mathrm{l}$ of the sample solution and incubated at $37^{\circ} \mathrm{C}$ for 30 minutes, before absorbance at $593 \mathrm{~nm}$ was measured. All the results were expressed as $\mu$ mol Trolox Equivalent (TE) per gram dry weight of the microalgae.

\subsubsection{Ferrous-Ion Chelating Assay}

The ferrous ion chelating assay was carried out according to Decker and Welch (1990). First, $100 \mu 1$ of the sample solution were added to $100 \mu \mathrm{l}$ of double-distilled water, followed by $25 \mu 1$ of $0.5 \mathrm{mM}$ ferrous chloride. Absorbance was measured at $550 \mathrm{~nm}$ before as well as 20 minutes (at room temperature) after $2.5 \mathrm{mM}$ of ferrozine (final concentration) was added. All the results were expressed as $\mu$ mol EDTA equivalent per gram dry weight of the microalgae.

\subsubsection{Determination of Total Phenolic Content}

The assay of the total phenolic content was carried out following Blaise, Owen, Stephen, Anthony \& Thomas (2009). The initial solution was prepared by mixing $300 \mu \mathrm{l}$ of $3 \% \mathrm{HCl}$ with $200 \mu \mathrm{l}$ of the sample extract. The prepared mixture was vortexed vigorously and left for 3 minutes. Then, $100 \mu l$ of the initial solution was added into $1000 \mu \mathrm{l}$ of $3 \%$ sodium bicarbonate. The new mixture was vortexed vigorously and left for 2 minutes before $20 \mu \mathrm{l}$ of the Folin-Ciocalteu reagent was added. The new solution was vortexed and left for 30 minutes at room temperature. After that, $200 \mu \mathrm{l}$ of the final solution was loaded into each well of a 96-well plate. All experiments were run in triplicates and the results were reported in $\mu \mathrm{mol}$ of gallic acid equivalents per one gram of dried extract.

\subsection{Statistical Analysis}

All the results were calculated as mean value \pm the standard deviation $(\mathrm{SD})(\mathrm{n}=3)$. A computer software statistical package for social sciences version 17.0 (SPSS Inc, IL, USA) was used to analyze the data. One way ANOVA and Duncan post-hoc tests were applied to test for significant differences at $p<0.05$. The correlation between antioxidant assay and total phenolic content was analyzed using the Pearson correlation test.

\section{Results and Discussion}

\subsection{DPPH Radical Scavenging Assay}

The radical-scavenging power could be easily determined by DPPH (Singh \& Rajini, 2004). Highly antioxidative substances will make the conversion from the purple chromogen radical ( $\left.\mathrm{DPPH}^{\bullet}\right)$ to the pale yellow hydrazine. Table 1 shows the antioxidant effects of the different-polarity solvents extracted. For the extract of Chaetoceros sp., chloroform extract showed the highest radicals-scavenging power among all of the solvents extracted with $106.68 \pm 0.91 \mu \mathrm{mol}$ TE per gram of dried extract. Although both chloroform and dichloromethane are very similar in chemical structure, the more powerful antioxidant substance was found in chloroform extract, which could scavenge the $\mathrm{DPPH}^{\bullet}$ radicals. As for extracts from Nannochloropsis sp., the dichloromethane extract showed the highest radical-scavenging power among all the solvents extracted, followed by the methanolic extract with $30.60 \pm 4.6$ and $23.18 \pm 0.6 \mu \mathrm{mol}$ TE per gram of dried extract, respectively. Following Hanaa, Hussein \& Gamal (2008), dichloromethane was used to extract carotenoid and tocopherol; thus, it is possible that the presence of these compounds contributed to the high scavenging power.

\subsection{Superoxide Anion Scavenging Assay}

A superoxide anion $\left(\mathrm{O}_{2}{ }^{\circ}\right)$ can be generated by a xanthine oxidase/hypoxanthine system: when the xanthine is catalyzed by xanthine oxidase, uric acid and superoxide radicals are also produced. Thus, superoxide reduces 
nitroblue tetrazolium (NBT) to blue formazon. Competition for superoxide occurs when an antioxidative substance is added with NBT (Lee, Jung, Seok, Kimb \& Yun, 2006). Superoxide itself does not cause much oxidative damage, but since it is an initiator in the radicals-production chain reaction, such as highly reactive hydroxyl radical $\left(\mathrm{HO}^{\circ}\right)$, peroxynitrate $\left(\mathrm{OONO}^{-}\right)$and singlet oxygen, it should be removed to indirectly reduce the number of radicals (Kim, \& Jeon, 2006). In this study, dichloromethane extract of Chaetoceros sp. showed remarkable superoxide scavenging power, at $1029.11 \pm 0.7 \mu \mathrm{mol} \mathrm{TE} / \mathrm{gram}$ of dried extract (Table 1), which might be due to the presence of tocopherol or carotenoid in the extract (Miller, Rice-Evans, Davies, Gopinathan \& Milner, 1993). The chloroform extract of Chaetoceros sp., which showed high scavenging power in the DPPH assay, failed to show any potential to scavenge superoxide radicals. On the other hand, the methanolic extract of Nannochloropsis sp. had a significantly higher superoxide scavenging power, at $3224.46 \pm 1.2 \mu \mathrm{mol} \mathrm{TE} / \mathrm{gram}$ of dried extract. Methanolic extract might contain unknown active compounds that effectively scavenged superoxide radicals. Identification of the compounds is needed for further evaluation.

\subsection{Ferric-Reducing Antioxidant Power (FRAP) Assay}

This assay is based on the expectation that ferric-reducing power is directly proportional to antioxidant capacity: in previous research, most ferric reducing agents also possessed antioxidative properties. Normally, ferrous ions do not reach extreme levels in any given biological system, due to the production of the highly reactive hydroxyl radical, from the Fenton reaction, when it reacts with hydrogen peroxide. Nevertheless, this indirect antioxidant determination assay at low $\mathrm{pH}$ levels is still widely in use. It is important to note here that not all reducing agents are antioxidative substances, or vise versa. Conversion of the ferric ion to the ferrous ion, which bonds to tripyridyltriazine to form $\mathrm{Fe}^{2+}$-TPTZ blue complex, can be measured at $593 \mathrm{~nm}$ (Benzie \& Strain, 1999). The limitation of this assay is that the SH-group containing the antioxidative substance cannot be measured (Ronald \& Guohua, 1999). Chloroform extract from Chaetoceros sp. achieved the most powerful reducing ability among all of the solvent extracts (Table 1). This result indicates that chloroform extract may contain most of the antioxidative substances in these algae. The dichloromethane extract of Nannochloropsis sp. attained the most powerful reducing ability, at $470.72 \pm 0.59 \mu \mathrm{mol}$ TE per gram of dried extract, which means that this extract may be rich in carotenoids with high antioxidant properties.

\subsection{Ferrous-Ion Chelating (FIC) Assay}

The ability to chelate off the ferrous ion is important to avoid a Fenton reaction, which would produce the most harmful radical, the hydroxyl radical. The production of the hydroxyl radical is a key initial step to producing other harmful radicals, which should be avoided. Indeed, ferrous ions may be released in the breakdown of red blood cells, causing the levels of ferrous ion in the body to increase (Prasad, Engelman, Jones \& Das, 1989). For Chaetoceros sp., the results showed that hexane extract had the most chelating ability, and that chloroform extract gave the least chelating power. This result suggested that most substances found in hexane extract contained the protonated form, one of the indicators of chelating properties, which was found least in chloroform extract. For Nannochloropsis sp., the methanol extract had the most chelating ability, and the dichloromethane and chloroform extracts did not have chelating power. Thus, methanol extract may contain some compounds that contain the protonated form that is highly needed for chelating properties.

\subsection{Determination of Total Phenolic Content}

The phenolic compound and its derivatives, including simple phenols, flavonoids, phenylproponoids, tannins, lignins and many other substances, contain aromatic rings and hydroxyl groups that will determine the radical scavenging power of the compound (Dziedziz \& Hudson, 1983). Many phenolic compounds have been reported as antioxidants, such as pheophytin from green microalgae (Nishibori \& Namiki, 1988) and phlorotannins from brown microalgae (Nagayama, Shibata, Fujimoto, Honjo \& Nakamura, 2003), but they might not be major contributors to the antioxidant capacities of microalgae ( $\mathrm{Li}$, Cheng, Wong, Fan, Chen, \& Jiang, 2007). In this study, the hexane extract of Chaetoceros sp. was found to have the highest total phenolic content. Surprisingly, the methanol extract from the same species showed negative results for phenolic content, but it showed high antioxidant capacities in other assays, indicating that the high antioxidant capacity is not related to the phenolic compounds, consistent with the study by Nagayama, Shibata, Fujimoto, Honjo \& Nakamura (2003). All of the solvent extracts from Nannochloropsis sp. showed relatively lower total phenolic content than the extract of Chaetoceros sp. (Fig 1).

\subsection{The correlation between antioxidant capacity and total phenolic content}

The correlation between the DPPH assay and the phenolic contents was found to be insignificant $\left(\mathrm{R}^{2}=0.144\right)$ (Fig.2). Both the NBT and the FRAP assay results showed an inverse correlation when plotted with the total phenolic content $\left(R^{2}=-0.296\right.$ and -0.306 , respectively). Only the FIC assay results significantly correlated $(p<$ 
$0.05)$ with the total phenolic content $\left(\mathrm{R}^{2}=0.804\right)$. This indicates that phenolic compounds might not be a major contributor to the antioxidant capacities for either of these microalgae. The fact that the microalgae contain various antioxidant compounds, such as carotenoids, polyunsaturated fatty acids and polysaccharides, further supports this observation (Mohamed, 2008; Holtin, Kuehnle, Rehbein, Schuler, Nicholson, \& Albert, 2009). Although phenolic compounds are found to be the major contributor to antioxidant activities in many higher-order species of such as plants, this might hold true for microalgae. Dixon \& Palva (1995) demonstrated that antioxidants such as phenolic compounds are in plants part of a complex defense mechanism against a wide range of stresses, and thus accumulate in response to these stresses. The microalgae in this study were cultured in controlled conditions, and thus might not have been exposed to stresses, causing fewer phenolic compounds to be produced, as compared to plants.

\section{Conclusion}

In this study, the antioxidant properties and total phenolic content of various extracts of a diatom, Chaetoceros sp., and a green microalgae, Nannochloropsis sp., were determined. Chaetoceros sp. extracts showed a higher antioxidant capacity $(\mathrm{p}<0.05)$ than Nannochloropsis sp. extracts did. The results also showed that different solvent extracts contained different antioxidant capacities in terms of reducing and radical-scavenging power. The correlation between the antioxidant properties and the total phenolic content was not significant $(\mathrm{p}>0.05)$, indicating that phenolic compounds might not be a major source of the antioxidant properties found in these two microalgae. Further study on other antioxidant compounds from these microalgae should be undertaken to confirm this observation.

\section{References}

Antonio, J. E., Isabel, J. J., Raquel, P., \& Fulgencio, S. C. (2001). Antioxidant activity of fresh and processed edible seaweeds. Journal of the Science of Food and Agriculture, 81, 530-534.

Aoronson, S., \& Dubinsky, Z. (1982). Mass production of microalgae. Experientia, 38, 36- 40.

Benzie, I. F. F., \& Strain, J. J. (1999). Ferric reducing / antioxidant power assay: direct measure of total antioxidant activity of biological fluids and modified version for simultaneous measurement of total antioxidant power and ascorbic acid concentration. Methods Enzymol Journal, 299, 15-27.

Blaise, W. L., Owen, K. D., Stephen, B., Anthony, D., \& Thomas D. (2009). Antioxidant activity of Sonoran Desert bee pollen. Food Chemistry, 115, 1299 - 1305.

Christophersen, A. G., Jun, H., Jorgensen, K., \& Skibsted, L. H. (1991). Photobleaching of astaxanthin and canthaxanthin: quantum-yields dependence of solvent, temperature, and wavelength of irradiation in relation to packaging and storage of carotenoid pigmented salmonoids. European Food Research and Technology, 192, 433-439.

Decker, E. A., \& Welch, B. (1990). Role of ferritin as a lipid oxidation catalyst in muscle food. Journal of Agriculture and Chemistry, 38, 674-677.

Dixon, R. A., \& Palva, N. (1995). Stress-induced phenylpropanoid metabolism, Plant Cell, 7, 1085-1097.

Dziedziz, S. Z., \& Hudson, B. J. F. (1983). Polyhydroxychalcones and flavanones as antioxidants for edible food. Food Chemistry, 12,205-212.

Gwo, J. C., Chiu, J. Y., Chou, C. C., \& Cheng, H. Y. (2005). Cryopreservation of a marine microalga, Nannochloropsis oculata (Eustigmatophyceae). Cryobiology, 50, 338-343.

Halliwell, B., \& Gutteridge, J. M. C. (1999). Free Radicals in Biology and Medicine. $3^{\text {rd }}$ edition. Oxford: Oxford University Press.

Hanaa, H. A. E., Hussein, M. M., \& Gamal, S. E. (2008). Algal extracts improve antioxidant defense abilities and salt tolerance of wheat plant irrigated with sea water. Electronic Journal of Environmental, Agriculture and Food Chemistry, 7(4), 2812-2832.

Holtin, K., Kuehnle, M., Rehbein, J., Schuler, P., Nicholson, G. \& Albert, K. (2009). Determination of astaxanthin and astaxanthin esters in the microalgae Haematococcus pluvialis by LC-(APCI)MS and characterization of predominant carotenoid isomers by NMR spectroscopy. Analytical and bioanalytical chemistry, 395(6), 1613-22

Katsumi, Y. (1997). Recent advances in microalgal bioscience in Japan, with special reference to utilization of biomass and metabolites: a review. Journal of Applied Phycology, 8, 487-502. 
Lee, J. Y., Yoon, J. W., Kim, C. T., \& Lim, S. T. (2004). Antioxidant activity of phenylpropanoid esters isolated and identified from Platycodon grandiflorum A. DC. Phytochemistry, 65, 3033-3039.

Lee, I. K., Jung, J. Y., Seok, S. J., Kimb, W. G., \& Yun, B. S. (2006). Free radical scavengers from the medicinal mushroom Inonotus xeranticus and their proposed biogenesis. Bioorganic \& Medicinal Chemistry Letters, 16, $5621-5624$.

Li, H. B., Cheng, K. W., Wong, C. C., Fan, K. W., Chen, F., \& Jiang, Y. (2007). Evaluation of antioxidant capacity and total phenolic content of different fractions of selected microalgae. Food Chemistry, 102:771-776.

Miller, N. J., Rice-Evans, C. A., Davies, M. J., Gopinathan, V., \& Milner, A. (1993). A novel method for measuring antioxidant capacity and its application to monitoring the antioxidant status in premature neonates. Clinical Science, 84, 407-412.

Mohamed, Z. A. (2008). Polysaccharides as a protective response against microcystin induced oxidative stress in Chlorella vulgaris and Scenedesmus quadricauda and their possible significance in the aquatic ecosystem. Ecotoxicology, 17, 504-516

Nagayama, K., Shibata, T., Fujimoto, K., Honjo, T., \& Nakamura, T. (2003). Algicidal effect of phlorotannin from brown alga Ecklonia kurome on red tide microalgae. Aquaculture, 218, 601-611.

Nishibori, S., \& Namiki, K. (1988). Antioxidative substances in the green fractions of the lipid of Aonori (Enteromorpha sp.). Journal of Home Economics of Japan, 39, 1173-1178.

Omidreza, F., Antonio, L., Rita, P., Giancarlo, M., \& Luciano, S. (2005). Evaluation of the antioxidant activity of flavonoids by ferric reducing antioxidant powerQ assay and cyclic voltammetry. Biochimica et Biophysica Acta, 1721, 174-184.

Prasad, M. R., Engelman, R. M., Jones, R. M., \& Das, D. K. (1989). Effect of oxyradicals on oxymyoglobin. Deoxygenation, haem removal and iron release. Biochemical Journal, 263,731-736.

Ronald, L. P., \& Guohua, C. (1999). In vivo total antioxidant capacity: comparison of different analytical methods. Free Radical Biology \& Medicine, 27(11/12), 1173-1181.

Saha, L., Lajis, N.H., Israf, D.A., Hamzah, A.S., Khozirah, S., Khamis, S., \& Syahida, A. (2004). Evaluation of antioxidant and nitric oxide inhibitory activities of selected Malaysian medicinal plants. Journal of Ethnopharmacology, 92, 263-267.

Singh, N., \& Rajini, P.S. (2004). Free radical scavenging activity of an aqueous extract of potato peel. Food Chemistry, 85, 611-616.

Takashi, K., Tomoko, H., \& Sayuri, M. (2006). Antioxidant properties of dried product of 'haba-nori', an edible brown alga, Petalonia binghamiae (J. Agaradh) Vinogradova. Food Chemistry, 98, 545-550.

Takashi, K., Makiko, T., Tomoko, H., \& Yoko, A. (2004). Antioxidant properties of dried 'kayamo-nori', a brown alga Scytosiphon lomentaria (Scytosiphonales, Phaeophyceae). Food Chemistry, 89, 617-622.

Tarozzi, A., Marchesi, A., Cantelli-Forti, G., \& Hrelia, P. (2004). Cold-storage affects antioxidant properties of apples in Caco-2 cells. American Society for Nutritional Science, 1105-1109.

Tolga, G., Yaşar, D., \& Şevket, G. (2003). Effects of light path lengths and initial culture density on the cultivation of Chaetoceros muelleri (Lemmermann, 1898). Aquaculture, 217, 431-436.

Yasantha, A., Kim K. N., \& Jeon, Y. J. (2006). Antiproliferative and antioxidant properties of an enzymatic hydrolysate from brown alga, Ecklonia cava. Food and Chemical Toxicology, 44, 1065-1074. 
Table 1. Evaluation of the antioxidant effects of different-polarity solvents extracted by different radical screening tests for Chaetoceros sp. and Nannochloropsis sp.

\begin{tabular}{|c|c|c|c|c|}
\hline Solvent Extraction/ & DPPH & NBT & FRAP & FIC \\
\hline Species & $\mu \mathrm{mol} \mathrm{TE} / \mathrm{g} \mathrm{DE}$ & $\mu \mathrm{mol} \mathrm{TE} / \mathrm{g} \mathrm{DE}$ & $\mu \mathrm{mol} \mathrm{TE} / \mathrm{g} \mathrm{DE}$ & $\begin{array}{c}\mu \mathrm{mol} \text { EDTA eq } \\
\mathrm{g} \mathrm{DE}\end{array}$ \\
\hline \multicolumn{5}{|l|}{ Hexane } \\
\hline$\overline{\text { Chaetoceros sp. }}$ & $31.81 \pm 0.81^{\mathrm{a}}$ & $652.23 \pm 0.79^{\mathrm{a}}$ & $171.54 \pm 2.56^{\mathrm{a}}$ & $82.39 \pm 0.81^{\mathrm{a}}$ \\
\hline Nannochloropsis sp. & $14.04 \pm 4.97^{\mathrm{b}}$ & $678.28 \pm 0.73^{\mathrm{a}}$ & $186.07 \pm 1.82^{\mathrm{ab}}$ & $15.70 \pm 0.20^{\mathrm{bc}}$ \\
\hline \multicolumn{5}{|l|}{ Dichloromethane } \\
\hline Chaetoceros sp. & $35.15 \pm 1.38^{\mathrm{ac}}$ & $1029.11 \pm 0.70^{\mathrm{b}}$ & $225.17 \pm 1.34^{\mathrm{b}}$ & $25.74 \pm 7.69^{b}$ \\
\hline Nannochloropsis sp. & $30.60 \pm 4.60^{\mathrm{a}}$ & - & $470.72 \pm 0.60^{c}$ & - \\
\hline \multicolumn{5}{|l|}{ Chloroform } \\
\hline$\overline{\text { Chaetoceros sp. }}$ & $106.68 \pm 0.91^{\mathrm{d}}$ & - & $609.80 \pm 0.55^{\mathrm{d}}$ & $3.25 \pm 2.08^{\mathrm{c}}$ \\
\hline Nannochloropsis sp. & $15.27 \pm 1.03^{\mathrm{b}}$ & $227.91 \pm 2.73^{c}$ & $321.22 \pm 0.74^{\mathrm{e}}$ & - \\
\hline \multicolumn{5}{|l|}{ Methanol } \\
\hline Chaetoceros sp. & $39.22 \pm 2.52^{\mathrm{c}}$ & $803.31 \pm 60.38^{\mathrm{a}}$ & $492.50 \pm 0.56^{c}$ & $16.71 \pm 3.68^{b c}$ \\
\hline Nannochloropsis sp. & $23.18 \pm 0.55^{\mathrm{e}}$ & $3224.49 \pm 1.20^{\mathrm{d}}$ & $221.16 \pm 0.90^{\mathrm{b}}$ & $24.049 \pm 5.62^{\mathrm{b}}$ \\
\hline
\end{tabular}

All results represent the mean \pm one standard deviation $(n=3)$

Within the same column, results followed by different letters are significantly different at $\mathrm{p}<0.05$

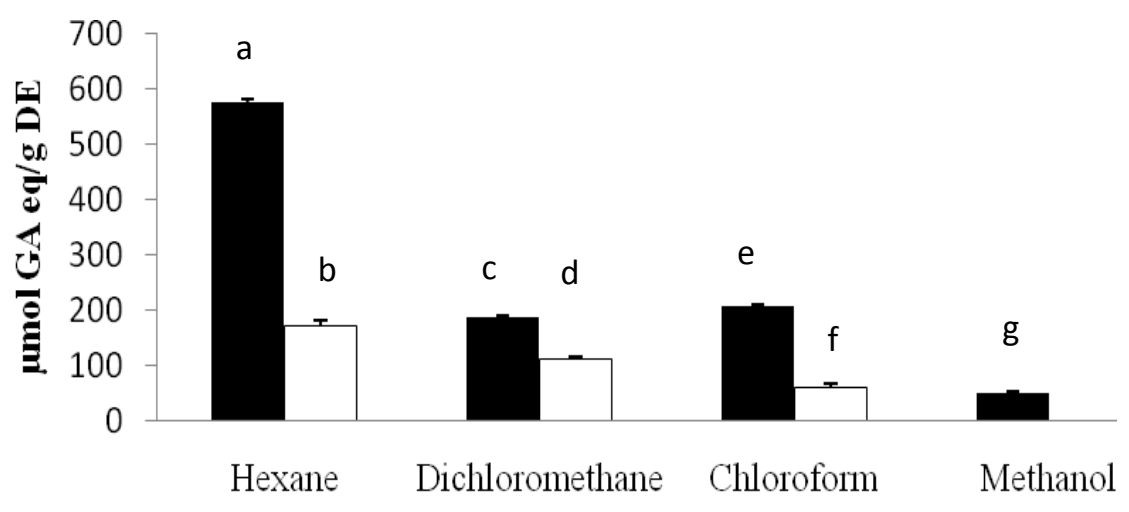

- Chaetoceros $\square$ Nannochloropsis

Figure 1. Total phenolic content of various extracts from the two microalgae 

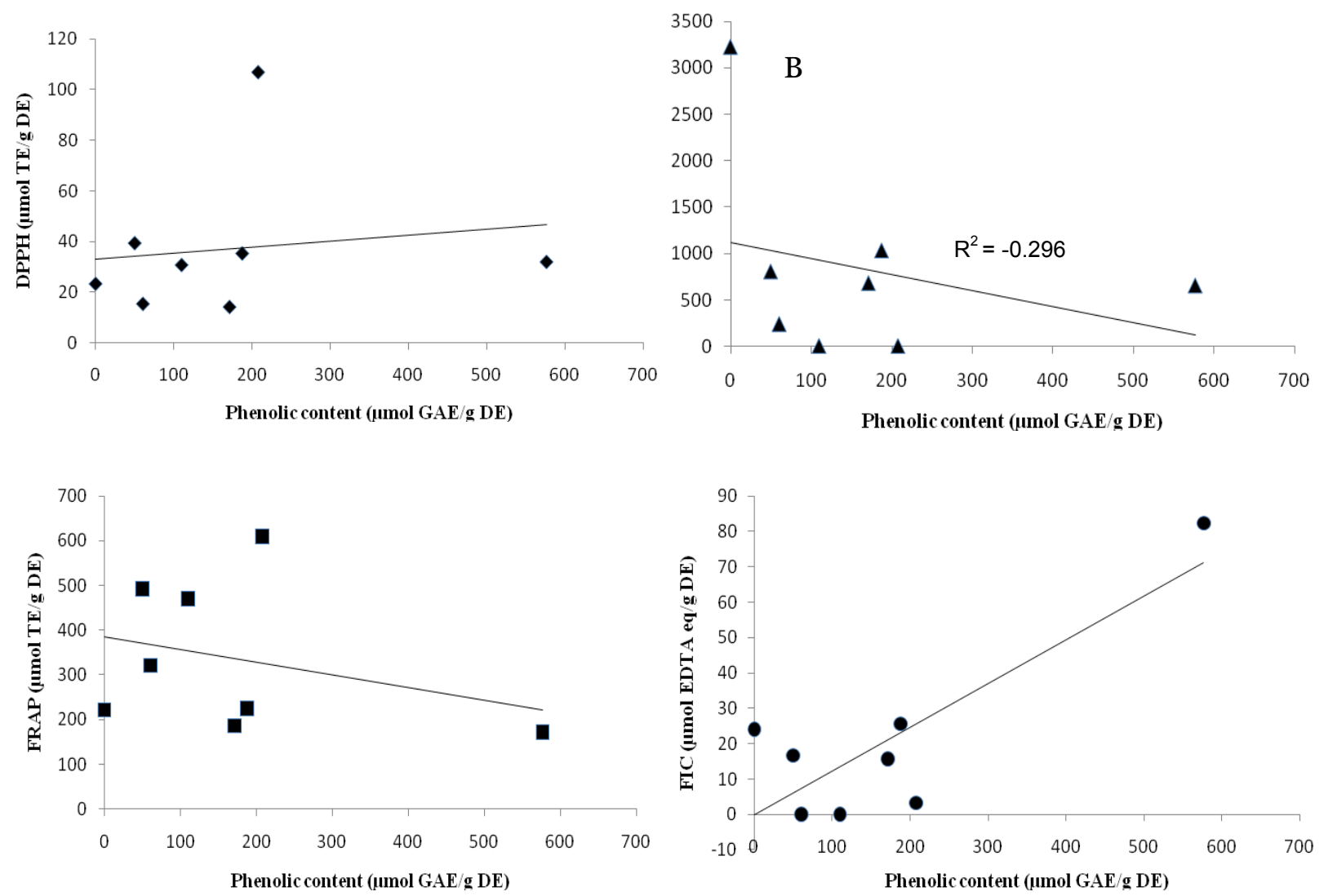

Figure 2. Correlation between phenolic content and (A) DPPH assay, (B) NBT assay, (C) FRAP assay and (D) FIC assay 